\title{
The External-Injection experiment at the SPARC_LAB facility
}

\author{
Andrea R. Rossi ${ }^{\mathrm{a}, *}$, Alberto Bacci ${ }^{\mathrm{a}}$, Marco Belleveglia ${ }^{\mathrm{b}}$, Enrica Chiadroni ${ }^{\mathrm{b}}$, \\ Alessandro Cianchi ${ }^{\mathrm{c}, \mathrm{b}}$, Giampiero Di Pirro ${ }^{\mathrm{b}}$, Massimo Ferrario ${ }^{\mathrm{b}}$, Alessandro Gallo ${ }^{\mathrm{b}}$, \\ Giancarlo Gatti ${ }^{\mathrm{b}}$, Cesare Maroli ${ }^{\mathrm{d}}$, Andrea Mostacci ${ }^{\mathrm{e}, \mathrm{b}}$, Vittoria Petrillo ${ }^{\mathrm{d}, \mathrm{a}}$, Luca Serafini ${ }^{\mathrm{a}}$, \\ Paolo Tomassini ${ }^{\mathrm{d}}$, Cristina Vaccarezza ${ }^{\mathrm{b}}$ \\ ${ }^{a}$ INFN - MI, via Celoria 16, 20133 Milan, Italy \\ ${ }^{\mathrm{b}}$ INFN - LNF, v.le E. Fermi, 00044 Frascati, Italy \\ c “Tor Vergata” University, Physics Department, via della Ricerca Scientifica 1, 00133 Rome, Italy \\ ${ }^{\mathrm{d}}$ University of Milan, Physics Department, via Celoria 16, 20133 Milan, Italy \\ e "La Sapienza" University, SBAI Department, via A. Scarpa 14, 00161 Rome, Italy
}

\section{A R T I C L E I N F O}

Available online 16 November 2013

Keywords:

Plasma acceleration

Plasma physics

Electron beam dynamics

\begin{abstract}
A B S T R A C T
At the SPARC_LAB facility of INFN-LNF we are installing a transport lines for ultra-short electron bunches and another for ultra-intense laser pulses, generated by the SPARC photo-injector and by the FLAME laser in a synchronized fashion at the tens of fs level, to co-propagate inside a hydrogen filled glass capillary, in order to perform acceleration of the electron bunch by a plasma wave driven by the laser pulse. The main aim of this experiment is to demonstrate that a high brightness electron beam can be accelerated by a plasma wave without any significant degradation of its quality. Motivations of the technical choices are made and expected performances are reported.
\end{abstract}

(c) 2013 Elsevier B.V. All rights reserved.

\section{Introduction}

The external injection experiment at SPARC_LAB aims at combining the high accelerating gradient characteristic of plasma-based accelerators with the production of high quality, stable and reproducible beams, typical of conventional RF linear accelerators, in order to overcome the formidable difficulties arising when exploiting the plasma-based acceleration schemes involving selfinjection, where any control on the bunch injection and acceleration is at most flimsy. In terms of electron beam parameters, the target consists in producing high brightness electron bunches that can eventually be employed in a variety of applications, such as front-end injectors for conventional accelerators (plasma-RF staging) and drivers for compact, short-pulse, radiation sources.

Plasma accelerators are based on the excitation of large amplitude waves (or wakes) in a plasma; they can be driven either by a high power laser pulses (for an extensive review, see Ref. [1]), in which case the technique is called Laser WakeField Acceleration (LWFA) or by particle bunches (first introduced by Ref. [2]), called Plasma WakeField Acceleration (PWFA). The driver first displaces plasma electrons while propagating in the plasma; the subsequent oscillation of the plasma creates a plasma wave (a wake) following the driver. The accelerating field of the wake depends on the

\footnotetext{
* Corresponding author.

E-mail address: andrea.rossi@mi.infn.it (A.R. Rossi).
}

unperturbed plasma density and the regime of the excited plasma wave and can reach a value typically up to $1 \mathrm{TV} / \mathrm{m}$ in the most non-linear regime.

\section{The SPARC_LAB facility}

The SPARC_LAB facility at LNF [3] consists in a conventional high brightness RF photo-injector, SPARC, and a multi-hundred terawatt laser, FLAME.

SPARC has been conceived to deliver high brightness electron beams up to $150 \mathrm{MeV}$. Its layout is peculiar, since the first two accelerating sections are equipped with additional focusing solenoids allowing to control transverse dynamics for attaining challenging phase space gymnastics, such as low energy RF bunch compression with the velocity bunching (VB) technique [4]. Profiting of such particular layout, electron beams with record brightness have been produced [5], carrying up to $1 \mathrm{kA}$ peak current with rms normalized emittance of about $1.5 \mathrm{~mm}$ mrad, to serve mainly the SASE and Seeded FEL experiments [6]. Moreover, SPARC demonstrated a novel active technique for beam generation and manipulation of ps-spaced, high brightness electron bunch trains, the so-called comb-beam [7], which allowed, together with VB [8], to produce high intensity coherent $\mathrm{THz}$ radiation [9] and will be used for driving a PWFA experiment [3]. 
The high power laser FLAME has been successfully put into operation with the achievement of its nominal specifications. A laser pulse carrying $6 \mathrm{~J}$ of energy, compressed down to about $30 \mathrm{fs}$ (FWHM) pulse length, has been transported into the experiment bunker. By focusing either into a supersonic gas jet or a solid target in the interaction chamber, it has produced self-injected bunches of electrons and proton beams. Such techniques are already known in literature, but the results confirm that all the different parts of the facility, from control to diagnostics, have been commissioned properly.

The integration of SPARC and FLAME is ongoing and particular care is being devoted to synchronization issues: both electrons and photons are as long as tens of fs and jitters typical of conventional $\mathrm{RF}$ timing may prevent the correct operation. For this reason a synchronization system based on optical distribution of the reference signal will be installed shortly. Preliminary results show that the jitters between the master oscillators can be as low as $10 \mathrm{fs}$ peak to peak. A conservative estimate of the overall effective time jitters is around 40-60 fs. A direct measurement of electron/ photons timing seems to be unfeasible; however, measuring the final energy spread of the accelerated electrons represents an extremely sensitive indirect estimate of timing [10].

A very versatile dogleg (Fig. 1) is under commissioning for delivering SPARC bunches to the external injection interaction chamber; this dogleg will also serve the Thomson back-scattering X-ray source planned to operate at SPARC_LAB [3].

\section{The external injection experiment}

In contrast to self-injection schemes without any induced injection mechanisms, external injection in LWFA presents many technical difficulties which need to be overcome; for example synchronization down to $10 \mathrm{fs}$ level is paramount to attain reproducibility (within $\approx 5 \%$ in term of electrons energy spread [10]) and efficient acceleration, while a precise laser/electrons alignment is needed for stability, high brightness and for avoiding damages to whatever laser guiding devices is eventually employed. For that reasons, and for avoiding unnecessary complications, at least at the beginning of the experiment, a judicious choice of all the experimental parameters is in need.

\subsection{Choice of parameters}

The external injection scheme is conditioned by the performances of existing RF technologies; in particular, the current limits in producing ultra-short bunches set the maximum value of the plasma wavelength $\lambda_{p}$ that can be exploited to post-accelerate the electron bunches. If the bunch length $\sigma_{z}$ is longer than a significant fraction of $\lambda_{p}$, the accelerated bunch could suffer from an excessive amount of energy spread, leading to an unacceptable emittance dilution when the electrons leave the plasma channel [15]. Since the peak accelerating electric field inside the plasma $E_{0}$ is proportional to $\lambda_{p}^{-1}$ and $\lambda_{p}^{-1} \propto n_{0}^{1 / 2}$, the constraint $\sigma_{z} \ll \lambda_{p}$ is also a constraint on the maximum accelerating gradient inside the plasma. Simulations show (see Section 3.2) that a safe plasma wavelength value is in the order of $100 \mu \mathrm{m}$, setting the plasma density to $n_{0} \approx 10^{17} \mathrm{~cm}^{-3}$ and the peak accelerating field $E_{0} \approx 30 \mathrm{GV} / \mathrm{m}$, which however would be attained only in the highly non-linear bubble regime. Increasing the plasma density could still be an option, since the prescribed value assumes a working point for the linac which is far from the limit; however, with increasing values of $E_{0}$ and decreasing values of $\lambda_{p}$, the whole process becomes more sensitive to jitters of whatever nature, advising against this option.

Plasma wave regimes can range from linear to highly nonlinear [16]: generally speaking, the first are more stable but yield less intense accelerating fields. The plasma wave charge density profile is a sinusoidal function in the longitudinal coordinate: this implies that the fields have non-linear (sinusoidal) behavior, contributing in increasing beam emittance. Conversely, the highly non-linear (bubble) regime is quite unstable but the wave charge density is an almost constant function of approximately spherical shape, yielding nearly linear, intense fields. Stronger fields mean a greater final energy but also a higher sensitivity to any jitter and on bunch mismatching. A good choice seems then to be right in the middle, exploiting a mildly non-linear wave, which corresponds to a condition on the laser parameter $a_{0} \approx 1$.

A major problem of the LWFA scheme is that, given a typical laser energy of a few Joules and a length of tens of fs, the condition $a_{0} \geq 1$ is met with a laser spot size $w_{0}$ of few tens of microns. The most intense accelerating fields are produced within a laser Rayleigh length, $l_{R}$, before and after the focus position, where $a_{0}$ is larger; we can then set the "effective" acceleration length equal to $2 l_{R}$. For Gaussian pulses with the aforementioned spot size, $l_{R}$ is at most of few centimeters, which is generally not enough to produce a significant increase of the electrons energy in the chosen working point. This problem can be solved by guiding the laser pulse over lengths which are usually much larger than the natural Rayleigh length. There are two main strategies for achieving guiding: either by transverse tapering of the plasma density $\left(n_{0} \propto r^{2}\right)$ or by using a capillary as an optical waveguide [11]. Transverse tapering, though harder to properly manage, has the advantage of preventing any laser energy leakage from the plasma channel, allowing a longer acceleration; moreover, since the laser pulse does not impinge on any solid surface, there are not concerns of damages. On the contrary, the capillary waveguide is easier to operate, although there are energy losses, due to the dielectric boundaries, and severely constrains the laser spot-size $w_{0}=0.645 R_{\text {cap }}$ ( $R_{\text {cap }}$ being the capillary inner radius) and focus position [12]. We opted for the capillary waveguide, at least

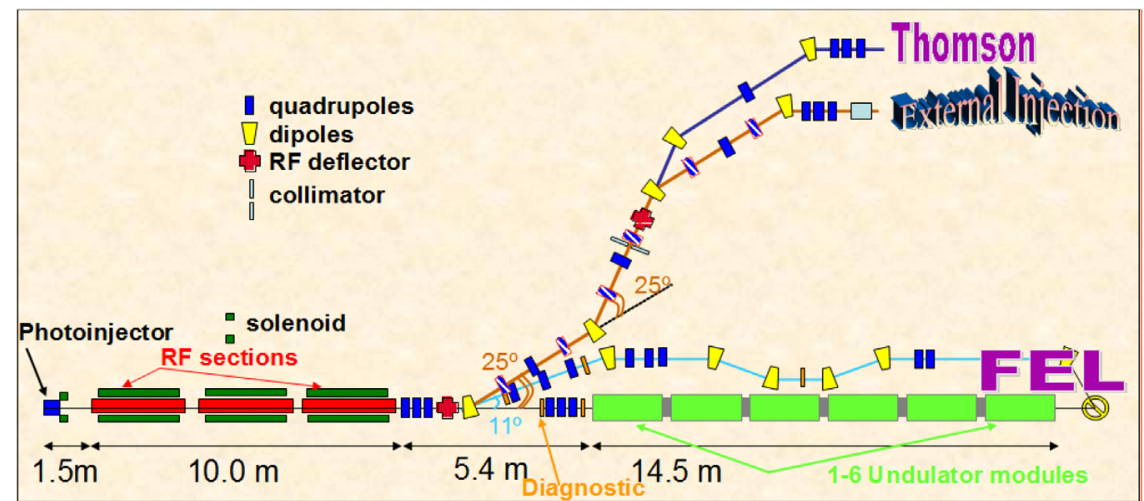

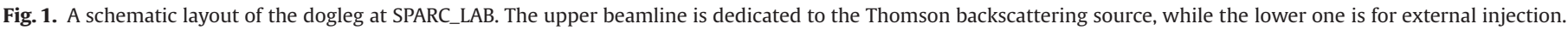


for the first part of the external injection experiment, due to its easy and cheap technical implementation. With this configuration we must also implement the constraint $\lambda_{p}<2 R_{\text {cap }}$, meaning that the "bulk" of the plasma wake shell not significantly interact with the guiding boundary; failing that, the behavior of the plasma wave cannot be easily predicted or simulated, since the plasma solid/ surface interaction is very complex and depend heavily on the plasma electrons energy. Moreover, since the matching of the laser pulse to the capillary is critical to perform a smooth propagation inside the waveguide, we should avoid any effect that could modify the laser waist dimension. In practice, this translate in an upper limit for $a_{0}$ that prevents the laser self-focusing [19].

For a proof of principle experiment, however, it can suffice an active accelerating length of order $l_{R}$, provided that the average energy increase achievable is larger than the energy spread due to (mainly temporal) jitters and the one induced by field curvature. For this reason, we will also consider a setting where the laser is not guided.

All the above-mentioned considerations are summarized in Fig. 2 (see Ref. [19] for details), assuming an incoming laser pulse energy of $3.5 \mathrm{~J}$ with a length of $35 \mathrm{fs}$, both of which are well within the possibilities of FLAME. In the plot, we restrained the laser parameter to be in the range $1.0 \leq a_{0} \leq 1.3$ in order to excite a mildly nonlinear wave and avoid self-focusing (white area between green lines); we also excluded the cavitation regime (area under the red line, see Ref. [19]) and the values of $n_{0}$ for which $\lambda_{p}<2 R_{\text {cap }}$ does not hold (area under the blue line). The pink dot represents the chosen working point: $R_{\text {cap }}=60 \mu \mathrm{m}$ and $n_{0}=10^{17} \mathrm{~cm}^{-3}$. This working point seems to be the best compromise between all the aforementioned conditions. Moreover, the resonant Lorentz factor, coming from the laser group velocity inside the plasma, turns out to be in excess of 90 , so that the dephasing length is more than $1.5 \mathrm{~m}$, much longer than the planned maximum capillary length allowed by the interaction chamber $(10-20 \mathrm{~cm})$. Moreover the value of $R_{\text {cap }}$ is such that the energy losses at the boundaries also have a characteristic length of over $1 \mathrm{~m}$.

\subsection{Start to end simulations}

Start-to-end simulations for the External-Injection experiment are performed using three different numerical codes: ASTRA [13] for the bunch generation at the photocathode and acceleration

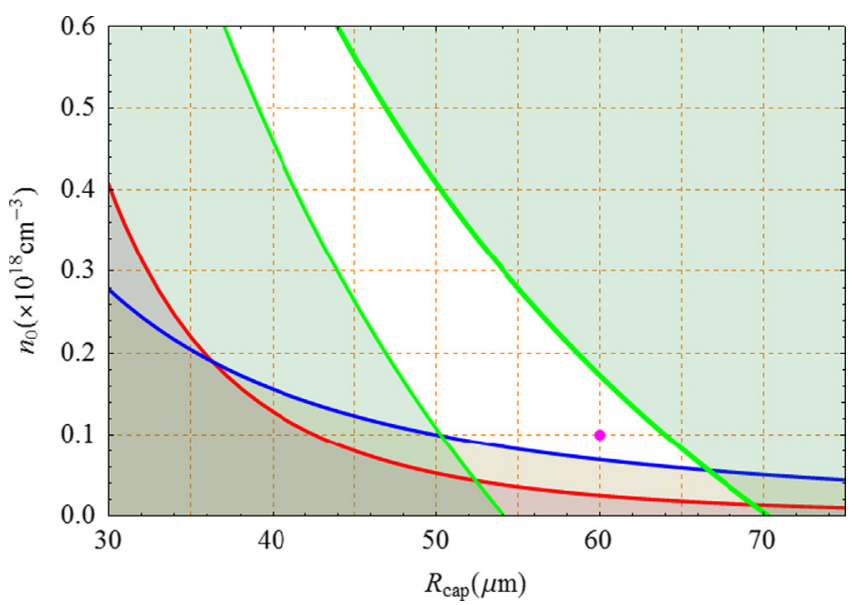

Fig. 2. Exclusion areas (colored) coming from physical and practical constraints; the white area enforces the $1<a_{0}<1.3$ condition (above the upper green line and below the lower), avoids cold wave breaking (above red line) and complies with the $\lambda_{p}>2 R_{\text {cap }}$ condition (above blue line). The chosen working point is shown by the pink dot. See text and Ref. [19] for details. (For interpretation of the references to color in this figure caption, the reader is referred to the web version of this paper.) down to the linac end, ELEGANT [14] for the transport inside the dogleg (see Fig. 1 for a schematic layout) and QFLUID2 for the acceleration in plasma.

QFLUID2 [10] is a numerical code which assumes cylindrical symmetry. The electron plasma component is treated as a fluid while the ions are a still background. The laser is Gaussian in both dimensions; its evolution is self-consistent and makes use of the envelope approximation. The injected bunch (macro) particles are fully kinetic and treated pretty much like in a particle in cell code, with the charge densities and currents deposed on the computational grid.

The electron bunch is extracted from the photocathode by a short, Gaussian laser pulse with an r.m.s. length $\sigma_{t}=300 \mathrm{fs}$ and compressed, by $\mathrm{VB}$, in the following traveling wave cavities, down to about $70 \mathrm{fs}$ with a final energy of $78 \mathrm{MeV}$. Afterwards it is again magnetically compressed during the transport in the dogleg by a factor of 2. The peak current associated to the bunch does never exceed a value of $300 \mathrm{~A}$, so that we do not expect significant effects from Coherent Synchrotron Radiation in the dogleg and from beam loading in the plasma. Even if beam loading can help in reducing the final energy spread (see, for example, Ref. [17], and references therein), in our mildly non-linear regime, it can also generate detrimental effects. In fact, since the plasma wake is not completely void of electrons, the electron bunch unavoidably acts as the driver of a secondary plasma wave, starting to loose some amount of energy to the plasma. The extent of this process is proportional to the beam current, so increasing it cannot be done without a further, detailed evaluation of pros and cons. This justifies the low value of the bunch charge $(\approx 20 \mathrm{pC})$, which also allows for a quite high overall longitudinal compression factor; lower charges, though possible, would require very expensive diagnostics systems. All the other choices done in setting up the acceleration, compression and transport parameters cope with the requirement of allowing a relatively easy operation of the machine. In Fig. 3 the transverse spot and the longitudinal phase space of the injected bunch are reported, together with a plot of the slice current. Such bunch still needs optimization; the position of the current peak should be moved in the head area of the beam itself in order to be in the region of the plasma wave where the transverse electric field is focusing (i.e. before the peak accelerating longitudinal field). Moreover the dimensions in the transverse spot and the two transverse phase spaces are quite different, with the bending $x$ plane having larger values both on $\sigma$ and on $\varepsilon_{\mathrm{n}}$. The insertion of one or more collimating slits before the interaction point seems to be needed in order to make the beam more symmetric.

\subsubsection{Acceleration without laser guiding}

We plan to perform the External Injection experiment in two steps of increasing difficulty. Step one will not make use of any guiding device for the laser pulse and constitutes a proof of principle of the external injection scheme. A gas cell will be employed only as a mean for confining the gas that will be ionized by the laser. In order to achieve a significant acceleration length, being the laser profile approximately Gaussian, we set the laser spot-size to $w_{0}=120 \mu \mathrm{m}$ so that twice the Rayleigh range is about $3 \mathrm{~cm}$, the length of the gas cell itself; with such parameters for the laser propagation, the value of $a_{0}$ ranges from 0.62 at injection up to 0.88 at the waist position, so that the excited plasma wave is almost linear. This regime could be useful to gain experience on the matching of the electron beam to the plasma channel focusing field.

The bunch resulting from the plasma acceleration is reported in Fig. 4, while the beam parameters are reported in Table 1 with a final energy of about $120 \mathrm{MeV}$, corresponding to an average 

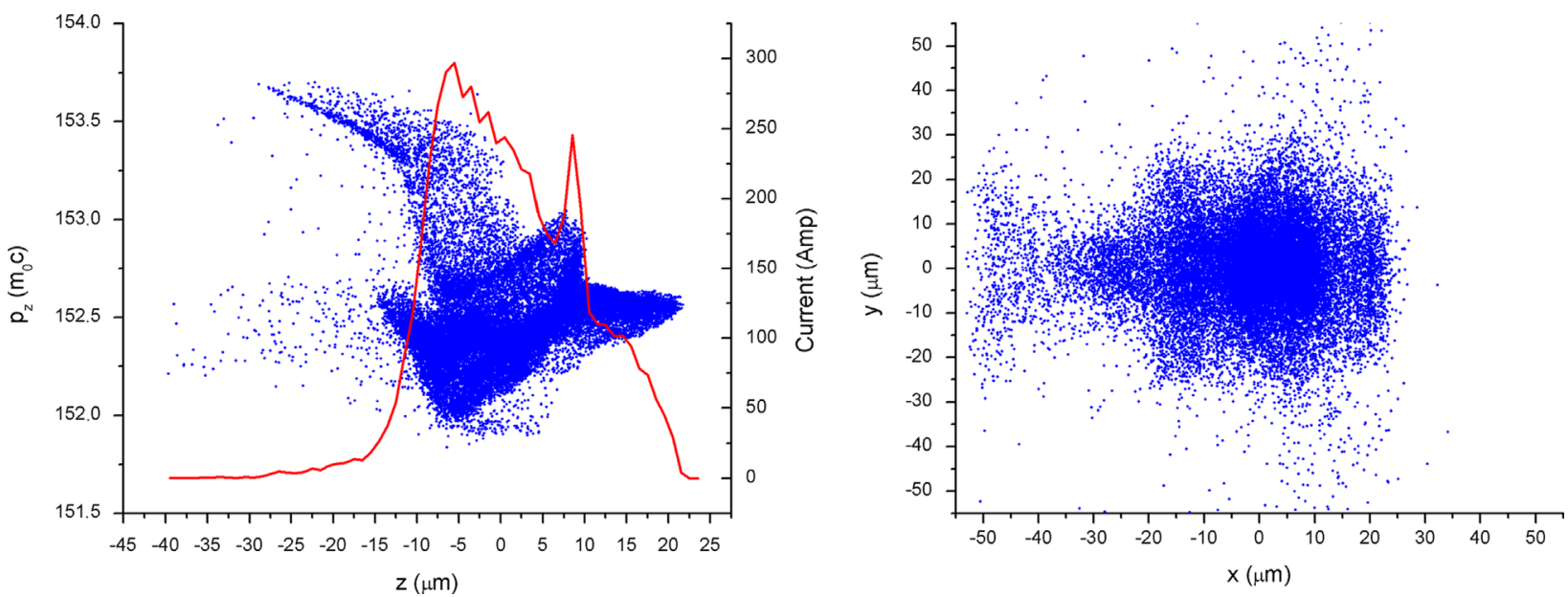

Fig. 3. Longitudinal phase space with slice current (left) and transverse spot (right) of the bunch before plasma acceleration.
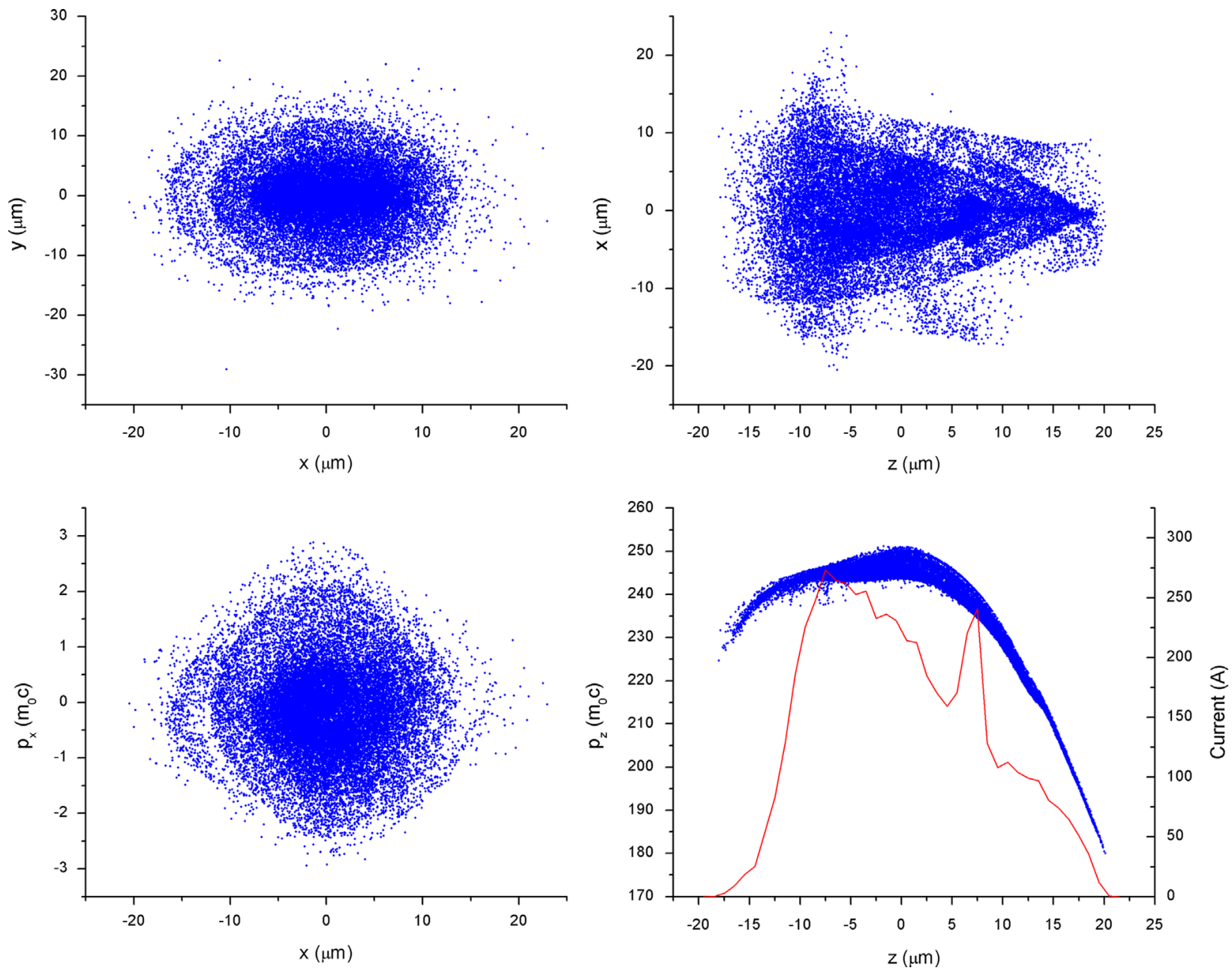

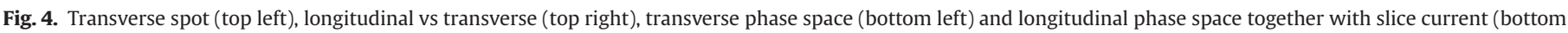
right) of the bunch after acceleration in the gas cell configuration.

accelerating field of $1.3 \mathrm{GV} / \mathrm{m}$. Since we work in an almost linear regime, some beam loading effects can be seen in the longitudinal phase space in correspondence of the current peaks. Moreover, the bunch is surrounded by a halo which corresponds to the asymmetric charge in the transverse spot of Fig. 3; such particles do contribute to the emittance value in Table 1 but would probably be lost to the beam pipe tube in the transport to the diagnostics station, so that the resulting emittance should be lower than what was reported. The presence of a transverse field depending on the longitudinal position can be clearly seen in the $x$ vs $z$ plot, where the bunch appears to have a triangular like shape. The peak current is less than the incoming bunch current since about $8 \%$ of the charge has been expelled from the accelerating bucket. 


\subsubsection{Acceleration with capillary guiding}

The goal of the second step of External Injection is to produce a high quality electron bunch. To this end, it is important to find a proper matching when the electrons enter the plasma and leave it. Analytic formulas do not apply since they are valid in the limiting situations of either linear waves or bubble regime. We then run many simulations assuming that we could change adiabatically the beam transverse size at the plasma entrance. Moreover, we sought the best performing value of the injection phase inside the plasma wave. The result was a delay from the laser pulse of $\Delta t=182 \mathrm{fs}$ as injection phase and the best matching was found to be a transverse size $\sigma_{x}=3.8 \mu \mathrm{m}$, as shown in Fig. 5, where the relative

Table 1

Beam parameters at different locations of the beamline. Positions: (1) is at extraction from the photocathode, (2) at the end of the linac, (3) at the end of the dogleg, (4) after plasma acceleration using a gas cell or (5) after plasma acceleration using a $10 \mathrm{~cm}$ capillary. Empty entries are either non-relevant or unchanged with respect to the previous value.

\begin{tabular}{lccccc}
\hline Position & $(1)$ & $(2)$ & $(3)$ & $(4)$ & $(5)$ \\
\hline Charge (pC) & 20 & & & 18.5 & 14 \\
$\sigma_{x}(\mu \mathrm{m})$ & 120 & 450 & 13 & 4.5 & 3.5 \\
$\sigma_{z}(\mathrm{fs})$ & 300 & 70 & 29 & & \\
Energy (MeV) & & 78 & & 120 & 630 \\
Energy spread (\%, uncorr.) & & 0.1 & 0.2 & $<1$ & 1 \\
$\varepsilon_{x \mathrm{n}}(\mathrm{mm}$ mrad) & & 0.23 & 2.7 & 4.5 & 3.5 \\
\hline
\end{tabular}

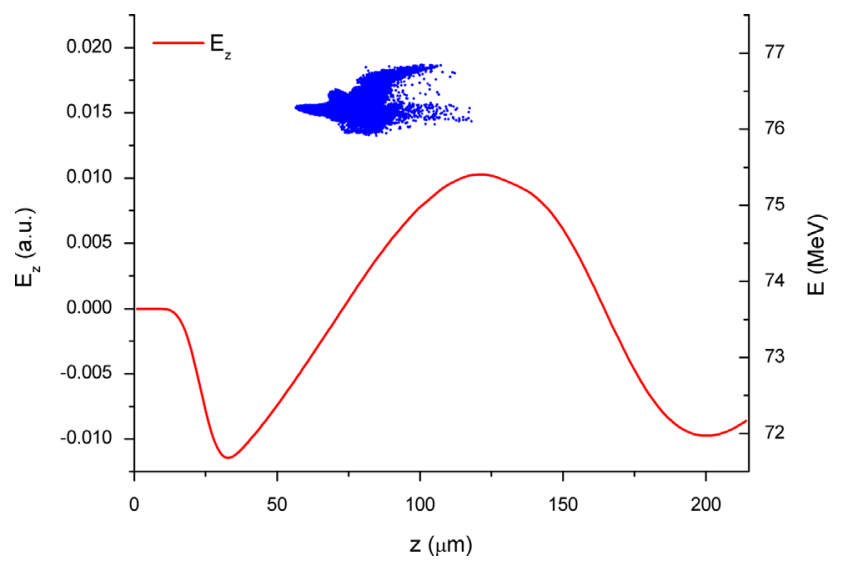

Fig. 5. Longitudinal electric field of the plasma wave (red line) together with the electron bunch longitudinal phase space (blue dots) at injection. Both are moving right to left. (For interpretation of the references to color in this figure caption, the reader is referred to the web version of this paper.) position between the bunch and the plasma wave (represented by its longitudinal electric field component) is displayed. Fig. 6 shows the beam size and emittance evolution along a $10 \mathrm{~cm}$ long capillary. This value represents only the maximum allowed capillary length that can be contained in the interaction chamber, which is, at present, under an advanced design stage.

The sudden, large increase of size and emittance soon after entering the plasma is due to the expulsion of the unmatched charge (the asymmetric particles in Fig. 3) from the accelerating plasma bucket. More charge is expelled as the acceleration process takes place due to the long wavelength (slow) oscillations of the envelope. These come from the laser driver: whenever a laser pulse is guided by hollow dielectric waveguide or a transverse plasma density tapering, its peak power value does oscillate [20] and the injected bunch experiences both transverse and longitudinal electric fields whose intensities vary with time. Together with the nonlinear dependence of the transverse field on the radial position, this contributes to emittance dilution and electrons expulsion. The fast oscillations, instead, are probably due to the longitudinal dependence of the fields, so that different beam slices (with different energy) experience betatron oscillations with different frequencies (different focusing field), adding up to the retrieved trend $[15,21]$. However, the bunch overall matching is quite good, since emittance increases only by about $30 \%$ of the initial value and the envelope, excluding the charge expulsion episodes, remains close to the shown qualitative matched envelope (blue line in Fig. 6) for linear focusing fields $\sigma_{x} \propto \gamma^{-1 / 4}$ [18].

The beam produced after the acceleration process is shown in Fig. 7 and its properties are summarized in Table 1. The table contains the relevant beam parameters in three positions before plasma acceleration (at photo-cathode, after linac and before interaction with plasma) and the bunch produced in the two configurations under consideration (gas cell and capillary). Contrary to the case of the gas cell configuration, there is no halo surrounding the beam and beam load does not play any significant effect. The scattered particles that can be seen in the trailing area of the longitudinal phase space are mainly due to the de-focusing nature of the transverse field in that region. They are also responsible for the high value of the slice emittance and energy spread, shown in Fig. 8. As said, moving the current peak in the head area of the beam would greatly improve performances. Moreover, with the mentioned injection parameters, the portion of expelled charge after the $10 \mathrm{~cm}$ capillary turns out to be about 28\%: this value will also be decreased by moving the current peak. The triangular shape of the $z, x$ projection of the bunch is again due to the longitudinal dependence of the transverse field. Such an undesired feature would be greatly reduced if the input bunch length could be reduced to the target value of $10 \mu \mathrm{m}$ FWHM. With such beam the value of energy spread could also be reduced well under the value reported in Table 1, as shown by Ref. [22].
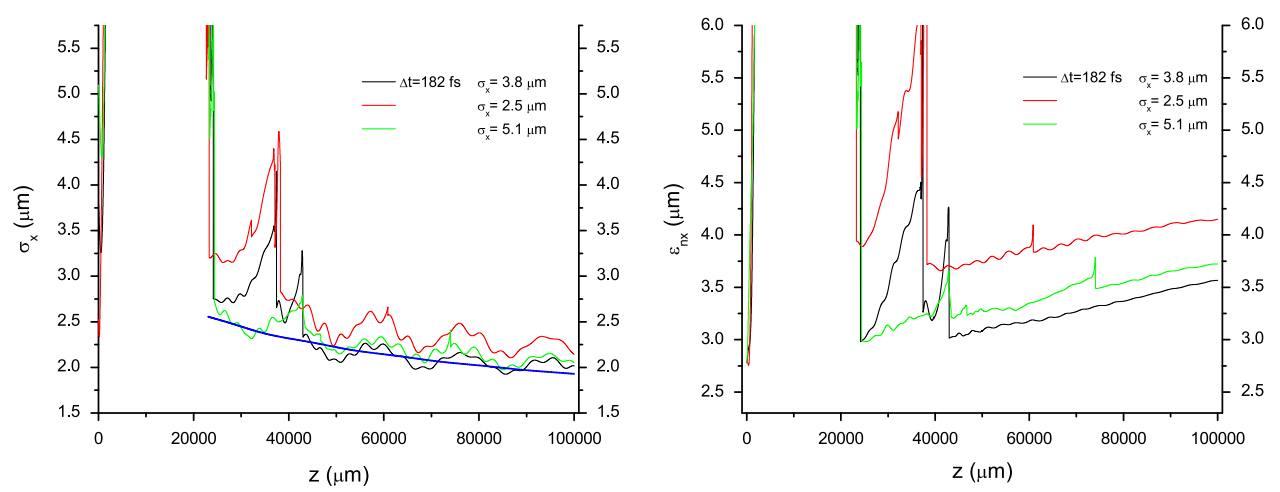

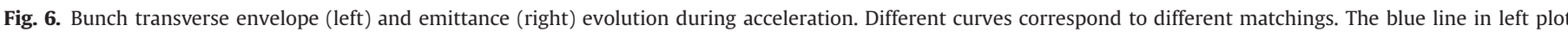

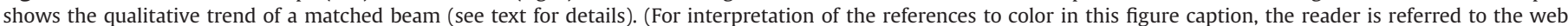
version of this paper.) 

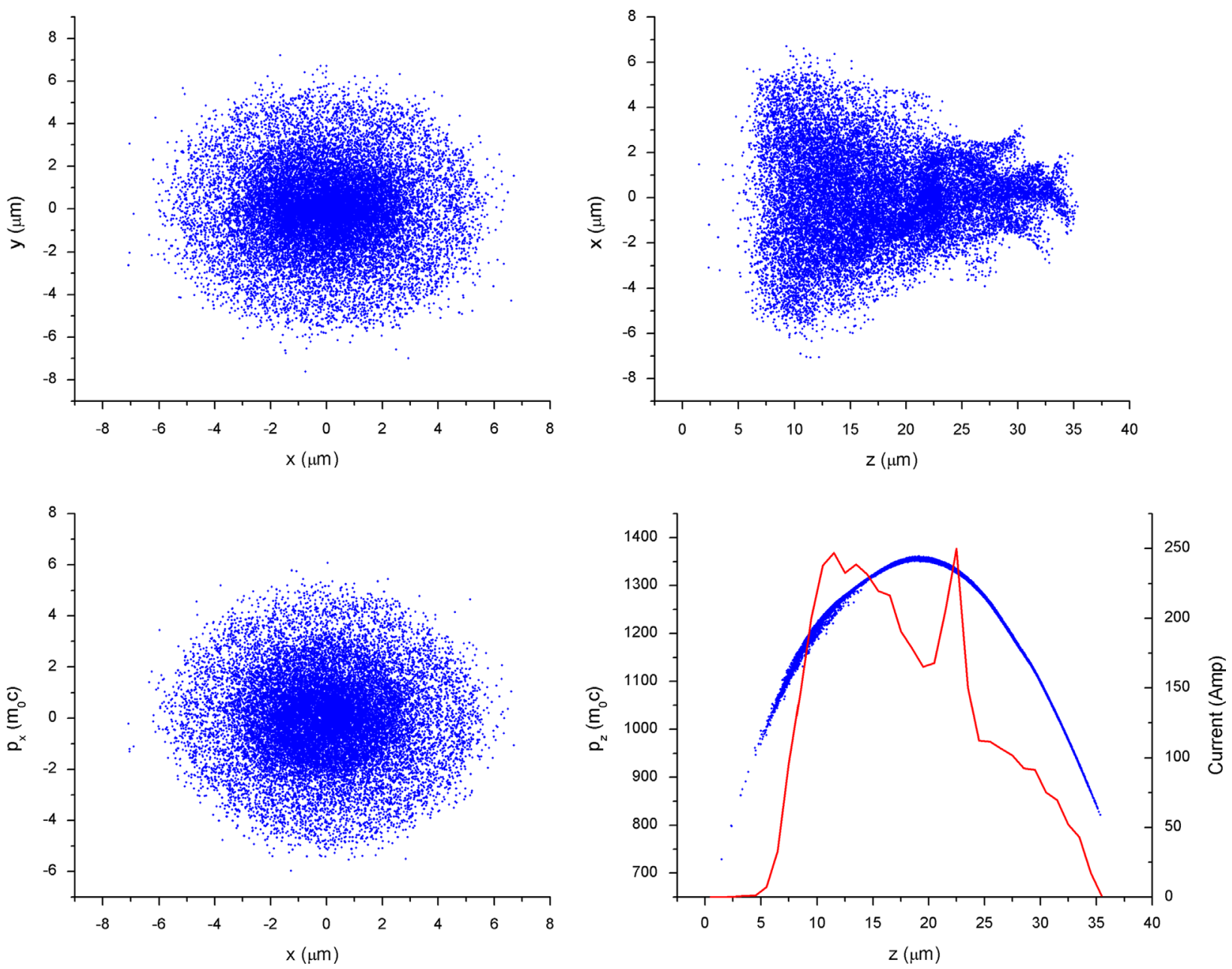

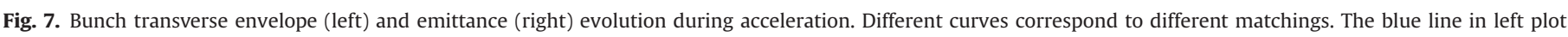

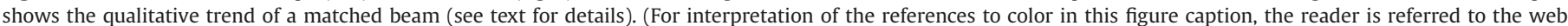
version of this paper.)

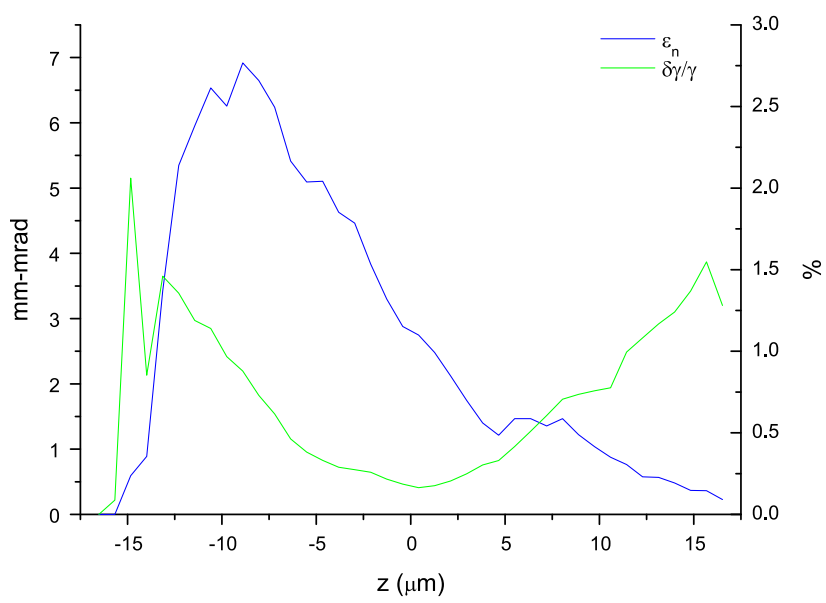

Fig. 8. Slice parameters of the bunch after acceleration in the capillary configuration.

\subsection{Diagnostics}

High accuracy and precision diagnostic tools are compulsory for both transverse and longitudinal characterization of the electron beam; preferably non-intercepting and single shot diagnostics should provide the required resolution of few tens of fs bunch length and few microns transverse beam size. More details on the diagnostics challenges posed by plasma accelerated beams can be found in Ref. [23]

At SPARC_LAB a wide energy range spectrometer will provide means of measuring the beam energy and its spread. The emittance will be measured by the quadrupole scan technique, tough, if the beam energy spread is relatively large, the results could be unreliable due to chromatic effects [24]. As for the longitudinal diagnostics, we plan to insert an RF deflector downstream the plasma interaction chamber. Finally, a device to measure the betatron radiation [25] and the transition radiation [26] is foreseen.

Diagnostics for the plasma wave appear to be quite difficult, due to the presence of the capillary, which prevents the use of methods such as shadowgraph to be employed. A possible technique [11], which allows to retrieve some information about the longitudinal properties of the plasma wave by measuring the spectrum of the outgoing laser pulse, is under consideration.

\subsection{Schedule}

The installation of the dedicated beam line is ongoing and will proceed alternating with regular machine operation till the end of 2014. The interaction chamber is in the final design stage and will be available before the end of the line installation. Commissioning 
will take place within the first half of 2015 and first plasma acceleration experiments are foreseen by the end of 2015 .

\section{Conclusions}

In this paper we showed the start to end simulation in preparation of the External Injection experiment at the SPARC_LAB facility of INFNLNF. Two different settings for the plasma acceleration have been considered: one easier, in which the laser pulse is not guided and acceleration occurs on the natural Rayleigh range of the laser itself, yielding an accelerated beam which can be considered as a proof of principle experiment; the other one foresees the exploitation of a hollow dielectric waveguide (glass capillary) in order to extend the useful accelerating length. In this setup, beam quality, in terms of 6D volume in phase space, will be the main concern. Simulations show a good result in that direction, although further optimization of the bunch generation and transport is still needed to reach optimal performances.

\section{Acknowledgments}

This work has been partially supported by the EU Commission in the Seventh Framework Program, Grant Agreement 312453 EuCARD-2 and by the Italian Ministry of Research in the framework of FIRB - Fondo per gli Investimenti della Ricerca di Base, Project no. RBFR12NK5K.

\section{References}

[1] E. Esarey, et al., Rev. Mod. Phys. 81 (2009).

[2] P. Chen, et al., Phys. Rev. Lett. 54 (1985) 693.

[3] M. Ferrario, et al., Nuc. Instrum. Methods Phys. Res. B 309 (2013) 183.

[4] L. Serafini, M. Ferrario, AIP Conf. Proc. 581 (2001) 87.

[5] A. Cianchi, et al., Phys. Rev. Spec. Top. Accel. Beams 11 (2008) 032801.

[6] L. Giannessi, et al., Phys. Rev. Lett. 106 (2011) 144801; M. Labat, et al., Phys. Rev. Lett. 107 (2011) 224801 ;

L. Giannessi, et al., Phys. Rev. Spec. Top. Accel. Beams 14 (2011) 060712.

[7] A. Mostacci, et al., in: Proceedings of the 2nd IPAC, THYB01, 2011.

[8] M. Ferrario, et al., Nucl. Instrum Methods Phys. Res. A 637 (2011) S43.

[9] E. Chiadroni, et al., Appl. Phys. Lett. 102 (2013) 094101.

[10] P. Tomassini, unpublished. Presentation available at <http://agenda.infn.it/ getFile.py/access? resId $=0 \&$ materialld $=$ slides $\&$ confld $=3444\rangle$.

11] F. Wojda, et al., Phys. Rev. E 80 (2009) 066403.

[12] N. Cross, et al., Phys. Rev. E 65 (2002) 026405.

[13] K. Flöttmann, 〈http://tesla.desy.de/ Ifroehli/astra/ >.

[14] M. Borland, Phys. Rev. Spec. Top. Accel. Beams 4 (2001) 070701.

[15] M. Migliorati, et al., Phys. Rev. Spec. Top. Accel. Beams 16 (2013) 011302; P. Antici, et al., J. Appl. Phys. 112 (2012) 044902.

[16] E. Esarey, C. Schroeder, W. Leemans, Rev. Mod. Phys. 81 (2009) 1229.

[17] M. Tzoufras, et al., Phys. Plasmas 16 (2009) 056705.

[18] M. Ferrario, in: Laser-Plasma Acceleration: Proceedings of the International School of Physics Enrico Fermi: Course 179. Varenna on Lake Como, Villa Monastero, 20-25 June 2011.

[19] P. Gibbon, in: Laser-Plasma Acceleration: Proceedings of the International School of Physics Enrico Fermi: Course 179. Varenna on Lake Como, Villa Monastero, 20-25 June 2011.

[20] N.E. Andreev, et al., Phys. Plasmas 9 (2002) 3999.

[21] T. Mehrling, et al., Phys. Rev. Spec. Top. Accel. Beams 15 (2012) 111303.

[22] A.R. Rossi, et al., in: Proceedings of the 3rd International Particle Accelerator Conference, New Orleans, Louisiana, USA, 2012, WEEPPB002.

[23] A. Cianchi, et al., Nucl. Instrum. Methods Phys. Res. A 720 (2013) 153.

[24] A. Mostacci, et al., Phys. Rev. Spec. Top. Accel. Beams 15 (2012) 082802.

[25] S. Corde, et al., Plasma Phys. Control. Fusion 54 (2012) 124023.

[26] E. Chiadroni, Ph.D. thesis, TESLAFEL2006-9. 